\title{
BMJ Open Intense Exercise for Survival among Men with Metastatic Castrate-Resistant Prostate Cancer (INTERVAL-GAP4): a multicentre, randomised, controlled phase III study protocol
}

\author{
Robert U Newton, ${ }^{1,2,3}$ Stacey A Kenfield, ${ }^{4}$ Nicolas H Hart, ${ }^{1,3,5}$ June M Chan,,${ }^{4,6}$ \\ Kerry S Courneya, ${ }^{1,7}$ James Catto, ${ }^{8}$ Stephen P Finn, ${ }^{9}$ Rosemary Greenwood, ${ }^{10}$ \\ Daniel C Hughes, ${ }^{11}$ Lorelei Mucci, ${ }^{12}$ Stephen R Plymate, ${ }^{13}$ Stephan F E Praet, ${ }^{13,14}$ \\ Emer M Guinan, ${ }^{15}$ Erin L Van Blarigan, ${ }^{6}$ Orla Casey,${ }^{15}$ Mark Buzza, ${ }^{16}$ Sam Gledhill, ${ }^{16}$ \\ Li Zhang, ${ }^{6,17}$ Daniel A Galvão, ${ }^{1,3}$ Charles J Ryan, ${ }^{4,17,18}$ Fred Saad $^{19}$
}

To cite: Newton RU,

Kenfield SA, Hart NH, et al. Intense Exercise for Survival among Men with Metastatic Castrate-Resistant Prostate Cancer (INTERVAL-GAP4): a multicentre, randomised, controlled phase III study protocol. BMJ Open 2018;8:e022899. doi:10.1136/ bmjopen-2018-022899

- Prepublication history for this paper is available online. To view these files, please visit the journal online (http://dx.doi org/10.1136/bmjopen-2018022899).

SAK and NHH contributed equally.

Received 14 March 2018 Accepted 4 April 2018

Check for updates

For numbered affiliations see end of article.

Correspondence to

Dr Nicolas H Hart;

n.hart@ecu.edu.au

\begin{abstract}
Introduction Preliminary evidence supports the beneficial role of physical activity on prostate cancer outcomes. This phase III randomised controlled trial (RCT) is designed to determine if supervised high-intensity aerobic and resistance exercise increases overall survival (OS) in patients with metastatic castrate-resistant prostate cancer (MCRPC). Methods and analysis Participants $(\mathrm{n}=866)$ must have histologically documented metastatic prostate cancer with evidence of progressive disease on androgen deprivation therapy (defined as mCRPC). Patients can be treatmentnaïve for $\mathrm{mCRPC}$ or on first-line androgen receptor-targeted therapy for mCRPC (ie, abiraterone or enzalutamide) without evidence of progression at enrolment, and with no prior chemotherapy for mCRPC. Patients will receive psychosocial support and will be randomly assigned (1:1) to either supervised exercise (high-intensity aerobic and resistance training) or self-directed exercise (provision of guidelines), stratified by treatment status and site. Exercise prescriptions will be tailored to each participant's fitness and morbidities. The primary endpoint is $\mathrm{OS}$. Secondary endpoints include time to disease progression, occurrence of a skeletal-related event or progression of pain, and degree of pain, opiate use, physical and emotional quality of life, and changes in metabolic biomarkers. An assessment of whether immune function, inflammation, dysregulation of insulin and energy metabolism, and androgen biomarkers are associated with $\mathrm{OS}$ will be performed, and whether they mediate the primary association between exercise and $\mathrm{OS}$ will also be investigated. This study will also establish a biobank for future biomarker discovery or validation.
\end{abstract}

Ethics and dissemination Validation of exercise as medicine and its mechanisms of action will create evidence to change clinical practice. Accordingly, outcomes of this RCT will be published in international, peer-reviewed journals, and presented at national and international conferences. Ethics approval was first obtained at Edith Cowan University (ID: 13236 NEWTON), with a further 10 investigator sites since receiving ethics approval, prior to activation.

Trial registration number NCT02730338.

\section{Strengths and limitations of this study}

- This is the first randomised controlled trial (RCT) to examine exercise and overall survival in men with prostate cancer.

- This is a novel multinational, multicentre and multidisciplinary RCT with 24 months of supervised tapered to self-managed exercise with behavioural and psychosocial support, compared with self-directed exercise with psychosocial support alone, in men with metastatic castrate-resistant prostate cancer (mCRPC).

- The study proposed will determine the efficacy of an individually tailored, progressive and autoregulated aerobic and resistance exercise programme, supervised by accredited exercise physiologists (or equivalent) in addition to usual medical care, in parallel with a health economics analysis to assess the health benefits, additional costs and potential savings of including exercise therapy as standard of care for men with MCRPC.

- The study has a translational team to investigate biomarkers associated with three candidate pathways: systemic inflammation, insulin/glucose metabolism and androgen biosynthesis; to study how they mediate the association between exercise and overall survival; and to establish a blood, urine and tissue biobank for future biomarker discovery or validation.

- The outcomes of this phase III RCT are limited to men with $\mathrm{MCRPC}$.

\section{INTRODUCTION}

An emerging body of literature supports the role of exercise during cancer treatment as a therapy which leads to improved outcomes, both in quality of life and potentially disease control. ${ }^{1}$ Identifying and evaluating 
low-toxicity adjuvant interventions, such as exercise, that can be combined with standard therapy to improve outcomes for men with prostate cancer is a high priority and has the potential to have a large impact on the clinical and public health burden of prostate cancer.

In 2006, Galvão et $a t^{2}$ reported that resistance exercise and programmes with resistance and aerobic exercise improved physical function and the quality of life in men without metastases on androgen deprivation therapy (ADT) for prostate cancer. These results were expanded in a subsequent report. Galvão $e t a l^{3}$ showed that combined resistance and aerobic exercise reversed the loss of muscle mass and improved the quality of life in patients with prostate cancer on ADT. In addition, Kenfield $e t a t^{t}$ reported that vigorous aerobic exercise after prostate cancer diagnosis was associated with a $60 \%$ lower risk of fatal prostate cancer and a $49 \%$ lower risk of all-cause mortality among men initially diagnosed with localised disease. The dose-specific effect of larger quantities of vigorous physical activity having greater survival benefit has also been reported by Friedenreich et al. ${ }^{5}$ In addition, one prospective study reported that resistance exercise was associated with a $33 \%$ lower risk of all-cause mortality in male and female cancer survivors while overall physical activity was not. ${ }^{6}$ These findings emphasise the potential benefits of exercise as an adjuvant treatment in prostate cancer. However, data on exercise and cancer survival to date have been from observational studies in which bias from confounding and reverse causation are of concern. Thus, a randomised controlled trial (RCT) is needed to test whether exercise, in particular higher intensity aerobic exercise and resistance exercise, impacts overall survival (OS) in men with prostate cancer. Additionally, treatment-related fatigue is a common side effect in men with advanced prostate cancer, and exercise may decrease fatigue and increase adherence to treatment regimens. ${ }^{7-9}$

While the prevailing view among patients and clinicians has been that exercise may be problematic for patients with cancer with advanced disease, recent research has demonstrated tailored resistance and aerobic exercise to be well tolerated, safe and effective for improving physical structure and function. ${ }^{10}$ Moreover, in patients with bone metastases, a highly tailored exercise prescription implementing a modular, multimodal approach and avoiding excessive loading of the skeletal lesions has been demonstrated to be safe and effective. ${ }^{10}$

There are many potential mechanisms by which exercise may lower risk of prostate cancer progression. ${ }^{111} 12$ Exercise influences all hormonal systems in the body, including key hormones relevant to prostate cancer, such as testosterone, growth hormone, insulin and insulin-like growth factor-1 (IGF-1). The androgen receptor (AR) and its transactivation by ligand are among the most important determinants of prostate cancer progression. Measurements of serum androgens (including its receptors and binding proteins) provide an important biomarker for the effectiveness of androgen deprivation and prostate cancer progression. The effects of exercise on serum androgen levels remain elusive to date, ${ }^{13}$ with current studies limited by low patient numbers and inadequate methods for measuring testosterone levels in the low ranges seen in men on ADT. ${ }^{14}$ This is especially true with the newer cyp17 inhibitors, such as abiraterone. Additionally, high levels of inflammatory biomarkers are associated with an increased risk of prostate cancer-specific mortality, ${ }^{15}$ and exercise is known to lower levels of circulating inflammatory biomarkers (eg, interleukin (IL)-6) in elderly populations. ${ }^{16}{ }^{17}$ Increased physical activity may also produce epigenetic modulations that may inhibit tumour cell proliferation, such as altering histone deacetylase pathways. Exercise and dietary changes may also lower cholesterol, which epidemiological studies have suggested are associated with decreased risk of prostate cancer and progression of prostate cancer. ${ }^{18}{ }^{19}$ Together, these observations suggest that exercise interventions with patients with prostate cancer may improve disease outcomes and quality of life. However, given the highly suggestive observational findings, an RCT is warranted to establish clear causal relationships and guide clinical recommendations.

The primary objective of the GAP4 Intense Exercise for Survival among Men with Metastatic Castrate-Resistant Prostate Cancer (INTERVAL-GAP4) study is to determine if high-intensity aerobic and resistance training plus psychosocial support increases OS compared with self-directed exercise (non-supervised exercise recommendations) plus psychosocial support, in patients with metastatic castrate-resistant prostate cancer (mCRPC). OS was chosen as the primary endpoint because it has clear biological, clinical and public health significance and is a validated endpoint for approval of new treatments among men with mCRPC. Additionally, OS data can be obtained with minimal loss to follow-up through review of medical and death records.

The secondary objectives are to compare time to disease progression, time to first occurrence of a symptomatic skeletal-related event (SSE), time to progression of pain, degree of pain, opiate use, physical and emotional quality of life, and change in levels of biomarkers of inflammation, energy metabolism and androgen metabolism between the supervised exercise and self-directed exercise groups. It will also be determined as to whether biomarkers of immune function, inflammation, energy metabolism and androgen metabolism are associated with OS among men with MCRPC, and the extent to which these biomarkers mediate the hypothesised association between high-intensity aerobic and resistance exercise and survival will be explored.

We hypothesise that men with MCRPC randomised to the supervised exercise arm will experience longer OS and time to disease progression, less SSE and progression of pain, less pain and opiate use, better physical function and quality of life, and more favourable levels of inflammatory, energy metabolism and other metabolic biomarkers compared with those in the self-directed exercise arm. 


\section{METHODS}

\section{Study design}

This is a multinational and multicentred, randomised controlled phase III clinical trial (INTERVAL-GAP4) recruiting 866 men with $\mathrm{mCRPC}$ to determine if supervised high-intensity aerobic and resistance training with psychosocial support increases OS compared with printed exercise recommendations (self-directed exercise) with psychosocial support. Patients will be randomly assigned
(1:1) to either supervised exercise or self-directed exercise following the provision of written informed consent, confirmation of clinical eligibility and successful completion of screening assessments (figure 1). This programme design has been chosen as it would be unethical to ask men with advanced prostate cancer to abstain from exercise for a 2-year period, owing to the documented health benefits of exercise in patients with prostate cancer with early-stage disease. Accordingly, men randomised to the

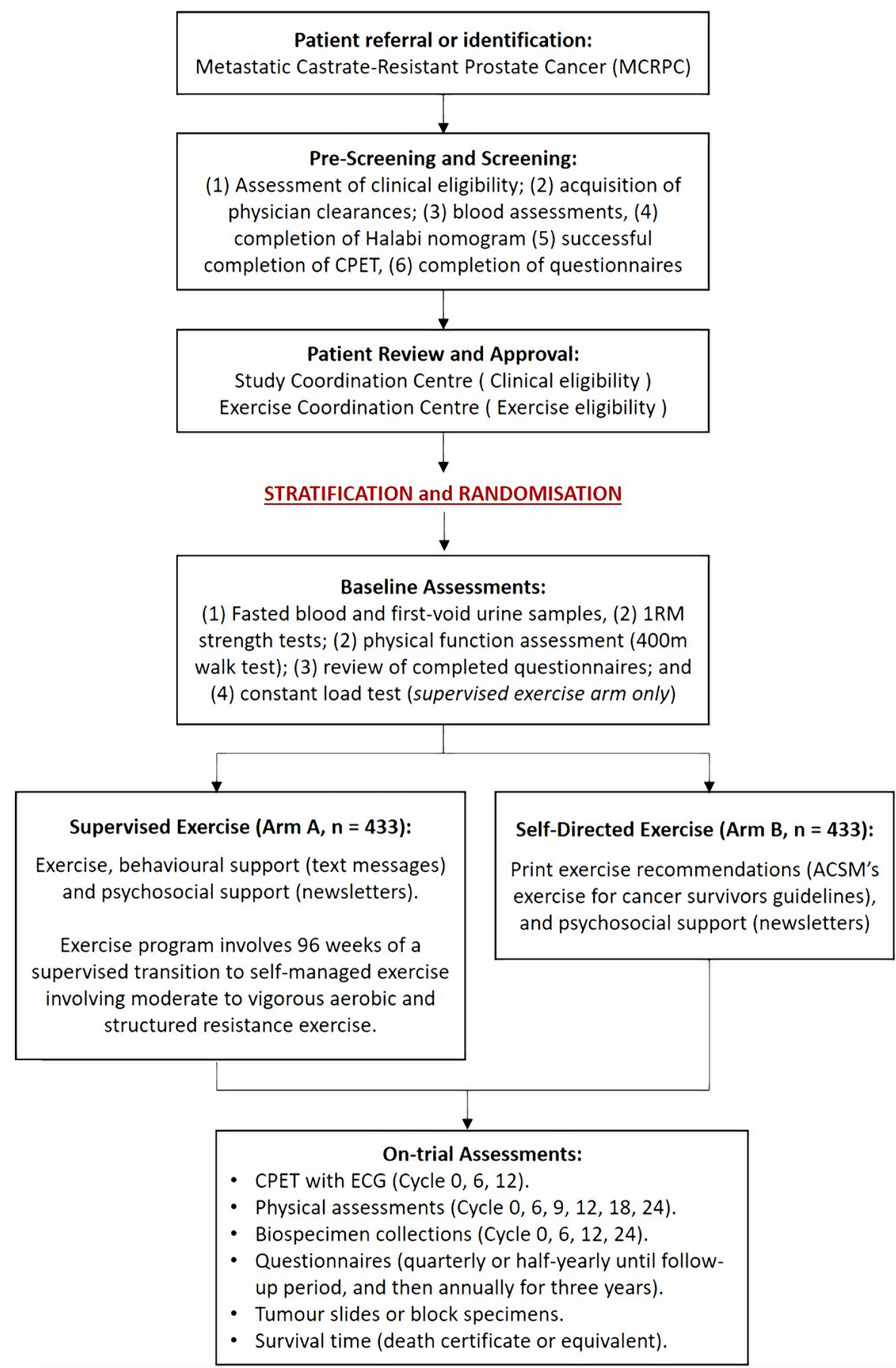

Figure 1 Schematic overview of the INTERVAL-GAP4 trial. 1RM, one-repetition maximum; ACSM, American College of Sports Medicine; CPET, cardiopulmonary exercise test; ECG, echocardiogram; INTERVAL-GAP4, Intense Exercise for Survival among Men with Metastatic Castrate-Resistant Prostate Cancer, Global Action Plan 4. 
control arm are free to engage in exercise under their own management (self-directed exercise), where changes in physical activity of both groups will be monitored.

The trial was prospectively registered on 10 March 2016 (https://clinicaltrials.gov/ct2/show/NCT02730338), prior to patient recruitment commencing, with the trial now recruiting.

\section{Participants}

Men with histologically documented adenocarcinoma of the prostate and progressive systemic metastatic disease despite castrate levels of testosterone $(<50 \mathrm{ng} / \mathrm{dL})$ due to orchiectomy or luteinising hormone releasing hormone (LHRH) agonist (defined as mCRPC), and who meet study inclusion (box 1) and exclusion (box 2) criteria will be recruited to the study. This patient population was chosen because the median OS among men with mCRPC is $32^{9}$ to $35^{8}$ months; thus, OS is a feasible outcome to examine within the budget and timeline of the proposed study when using patients with MCRPC as the target population. At enrolment, patients can be either treatment-naïve for mCRPC or on first-line AR-targeted therapy for mCRPC (ie, abiraterone or enzalutamide) without evidence of progression. Patients will be required to remain on $\mathrm{ADT}$ with a GnRH agonist/antagonist for the duration of their involvement in the study or have had prior bilateral orchiectomy. At enrolment, patients may have received chemotherapy for hormone-sensitive stages of the disease. Patients cannot have received chemotherapy for castrate-resistance status at enrolment. Patients are not permitted to be on any experimental therapies at enrolment; however, patients may be treated with chemotherapy or any other therapies for mCRPC postenrolment and randomisation.

\section{Screening}

Once referred to the trial through patients' managing clinician, and consented to the trial by an independent research officer, patients will undergo a screening process to confirm eligibility, with baseline measures taken prior to randomisation and completion of baseline exercise testing, if eligible. Measures necessary to complete the multivariable nomogram (http://www.cancer.duke.edu/ Nomogram/firstlinechemotherapy.html $)^{20}$ risk assessment are mandatory, including the presence of nodal, bone and/or visceral metastases; the use of opioid analgesics; Eastern Cooperative Oncology Group performance status (must be $\leq 1$ ); and the collection of standard-ofcare pathology (lactate dehydrogenase (LDH), albumin, haemoglobin, alkaline phosphatase (ALP), prostate-specific antigen) within 28 days prior to baseline assessments. Patients must have a Halabi nomogram risk of low or intermediate $(<195)^{20}$ to confirm clinical eligibility prior to attempting a symptom-limited, medically supervised cardiopulmonary exercise test (CPET) with echocardiogram (ECG) recording.

Patients who are currently participating in vigorous aerobic activity ( $>60 \mathrm{~min}$ per week) and/or structured

\section{Box 1 Inclusion criteria}

- Histologically documented adenocarcinoma of the prostate with progressive systemic metastatic disease, despite castrate levels of testosterone $(<50 \mathrm{ng} / \mathrm{dL})$. Castrate levels of testosterone must be maintained while on study.

- At enrolment, patients may be clinically eligible through two pathways:

- Pathway A: currently on abiraterone and/or enzalutamide and not progressing.

- Pathway B: preabiraterone and pre-enzalutamide with progressive disease.

- Progressive disease must be demonstrated by one or more of the following criteria:

1. Measurable disease progression:

$->20 \%$ increase in the sum of diameters of measurable lesions from the time of maximal regression; or the appearance of one or more new nodal, visceral or skeletal lesions.

2. Bone scan progression:

Appearance of one or more new lesions on a bone scan that is attributable to prostate cancer.

3. Prostate-specific antigen (PSA) progression:

- $P S A \geq 2 \mathrm{ng} / \mathrm{mL}$ that has risen serially on at least two occasions, each at least 1 week apart (PSA1<PSA2<PSA3).

- Patients must be on androgen deprivation therapy (ADT) with a gonadotropin-releasing hormine (GnRH) agonist/antagonistor prior bilateral orchiectomy. All patients are required to be on ADT during the study period.

- Receive a Halabi nomogram score $<195,{ }^{20}$ classified as low or intermediate risk.

- Eastern Cooperative Oncology Group performance status score $\leq 1$.

$\rightarrow$ Age $\geq 18$ years.

- Be willing to travel to one of the exercise facilities for exercise testing and training.

- Be willing to use the technological aspects of the trial for patient monitoring and support.

- Fluent in the language designated by the institution where the patient will be enrolled.

- No major surgery $\leq 4$ weeks at enrolment and fully recovered from any prior surgery.

- Medical clearance to complete a symptom-limited cardiopulmonary exercise test (CPET) with electrocardiography, and to complete a structured and progressive resistance and aerobic exercise programme of moderate-to-vigorous intensity.

- Must pass the CPET performed at screening (pre-enrolment), judged as achieving a rating of perceived exertion $\geq 9$ on the 10-point Borg Scale with no detected cardiac abnormalities. Patients with any abnormalities noted are permitted to enrol following cardiologist review and clearance.

- Meet the required baseline laboratory values: absolute neutrophil count (ANC) $\geq 1.5 \underline{x} 10^{9} / \mathrm{L}\left(\underline{1500 / \mu \mathrm{L}) ;}\right.$ platelet count $\geq 100 \times 10^{9} / \mathrm{L}(\geqq 100000 /$ $\underline{\mu L}$; creatinine $\leq 1.5 \times$ upper limits of normal; bilirubin $\leq 1.5 \times$ upper limits of normal; aspartate aminotransferase (AST) $\leq 1.5 \times$ upper limits of normal; and serum testosterone $\leq 50 \mathrm{ng} / \mathrm{dL}$.

- Patients with bone metastases must be cleared by the Exercise Coordination Centre following review of their most recent bone scan to ensure they are able to participate in most exercises required by the trial.

resistance training ( $\geq 2$ days per week) will be excluded. Patients must have no known contraindications to high-intensity aerobic or resistance exercise as determined by 


\section{Box 2 Exclusion criteria}

No previous progression while on treatment with abiraterone and/ or enzalutamide.

- No prior chemotherapy for metastatic castrate-resistant prostate cancer.

- Not currently receiving experimental treatment with non-approved drugs at enrolment.

- No known brain metastases.

- No known spinal cord compression, compromise or instrumentation due to metastatic disease. Radiation therapy for metastatic disease is allowed.

- No moderate to severe bone pain (Common Terminology Criteria for Adverse Events (CTCAE) V.5.0 grading criteria).

- No history of hypertension that is not well controlled.

- No congestive heart failure.

- No recent serious cardiovascular events (within 12 months), including but not limited to, transient ischaemic attack, cerebrovascular accident or myocardial infarction.

- No medical condition, such as uncontrolled infection or cardiac disease, which in the opinion of the relevant physician would make this protocol unreasonably hazardous for the patient.

- No serious or non-healing wound, ulcer or bone fracture.

- Not experiencing shortness of breath, chest discomfort or palpitations when performing activities of daily living.

- Does not have chest pain generated by physical activity and has not developed chest pain in the previous month.

- No peripheral neuropathy grade $\geq 3$ (CTCAE V.5.0 grading criteria).

- No psychiatric illness.

- No small cell neuroendocrine tumours or pure small cell carcinoma of the prostate.

- No current active second malignancy other than non-melanoma skin cancer.

- Not participating in vigorous aerobic exercise for more than $60 \mathrm{~min}$ per week.

- Not participating in structured resistance exercise for $\geq 2$ sessions per week.

their physicians. Following medical clearance, patients will be required to complete a series of baseline questionnaires and will attempt the symptom-limited CPET with ECG using a stationary, electronically braked cycle ergometer. Patients who successfully pass their CPET (ie, no cardiac abnormalities while achieving a maximal rating of perceived exertion (RPE $\geq 9$ of the 10-point Borg Scale)) will have their study information reviewed by the Exercise Coordination Centre (ECC; Edith Cowan University, Perth, Australia) for suitability of exercise prescription within the INTERVAL-GAP4 programme, with consideration given to the location, number and severity of bone metastases; and the Study Coordination Centre (SCC; University of California San Francisco (UCSF), California, USA) to confirm all clinical and study eligibility requirements prior to randomisation and subsequent baseline testing.

\section{Randomisation}

Patients will be centrally randomised by the SCC in a ratio of $1: 1$ to the two study arms, using block randomisation in random blocks of 2, 4 and 6 , and stratified by site and treatment status (ie, abiraterone or enzalutamide, yes/no; and radium-223, yes/no) as these therapies have a proven effect on progression-free survival. ${ }^{89} \mathrm{~A}$ research officer at the SCC with no patient contact will be responsible for uploading the randomisation schedule into the Research Electronic Data Capture (REDCap; a secure application for building and managing online surveys and databases). Site-based research coordinators will subsequently randomise patients through the REDCap system once approval from the SCC is received. Patients will not be informed of their group allocation until after the completion of their baseline visit to maintain the integrity of effort and the results of assessments performed. At the baseline visit, participants will complete all remaining assessments including physical function tests (ie, strength tests and $400 \mathrm{~m}$ walk test), fasting blood and first-void urine collection (ie, for correlative studies and biorepository storage), and any remaining questionnaires. Patients randomised to the supervised exercise arm will be enrolled into an automated text messaging programme to provide behavioural support, and participants in both arms will commence an automated newsletter education programme, circulated at the beginning of each cycle, intended to provide psychosocial support and enhance quality of life.

\section{Outcomes}

\section{Measurements}

This trial comprised a 96-week on-treatment period, approximating 2 years ( 24 cycles with each cycle spanning 28 days), followed by a 3-year follow-up period. Assessments are conducted at baseline and at routine intervals throughout the on-treatment period (table 1). After the on-treatment phase ends, patients will enter the follow-up phase of the trial, where their medical records and death certificates will be reviewed quarterly to quantify the primary endpoint of OS and prespecified secondary endpoints.

\section{Primary endpoint}

Overall survival

OS is the primary outcome of this RCT. It is a validated endpoint for the approval of new treatments in medicine, and feasible within the budget and timeline of the study as men with mCRPC have a median survival of 32-35 months. ${ }^{89}$ Patients will be followed for death a minimum of 36 months after randomisation. OS will be measured from the time of randomisation until death. Medical records and death certificates will be reviewed every 3 months to obtain survival status. Country-specific mortality databases will also be searched annually; cause of death will be determined through review of medical and death records. Importantly, quantification of OS through review of medical records and death certificates reduces loss to follow-up and missing data.

\section{Secondary endpoints}

Disease progression

Disease progression will be examined through review of patient medical records every six cycles and measured 


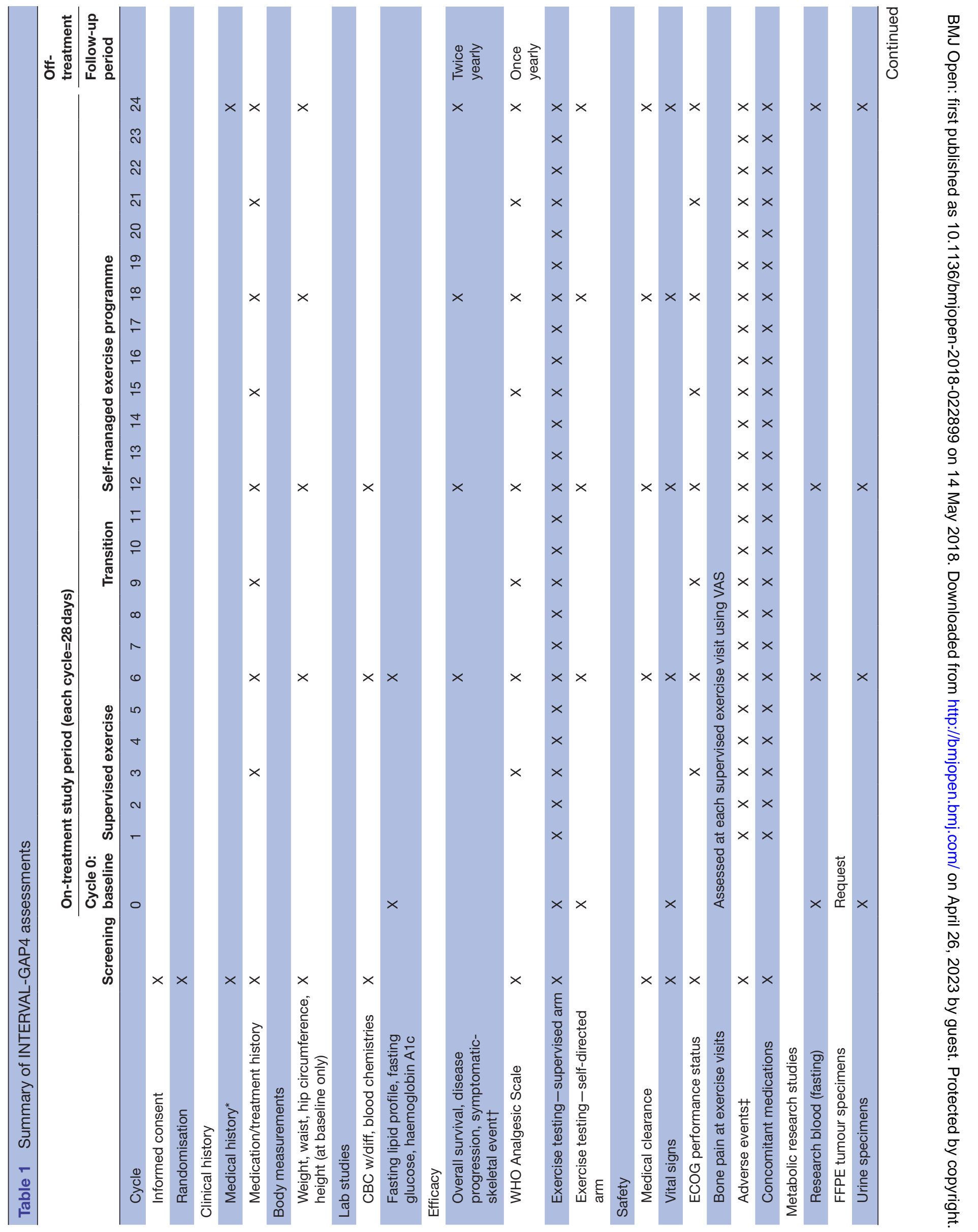



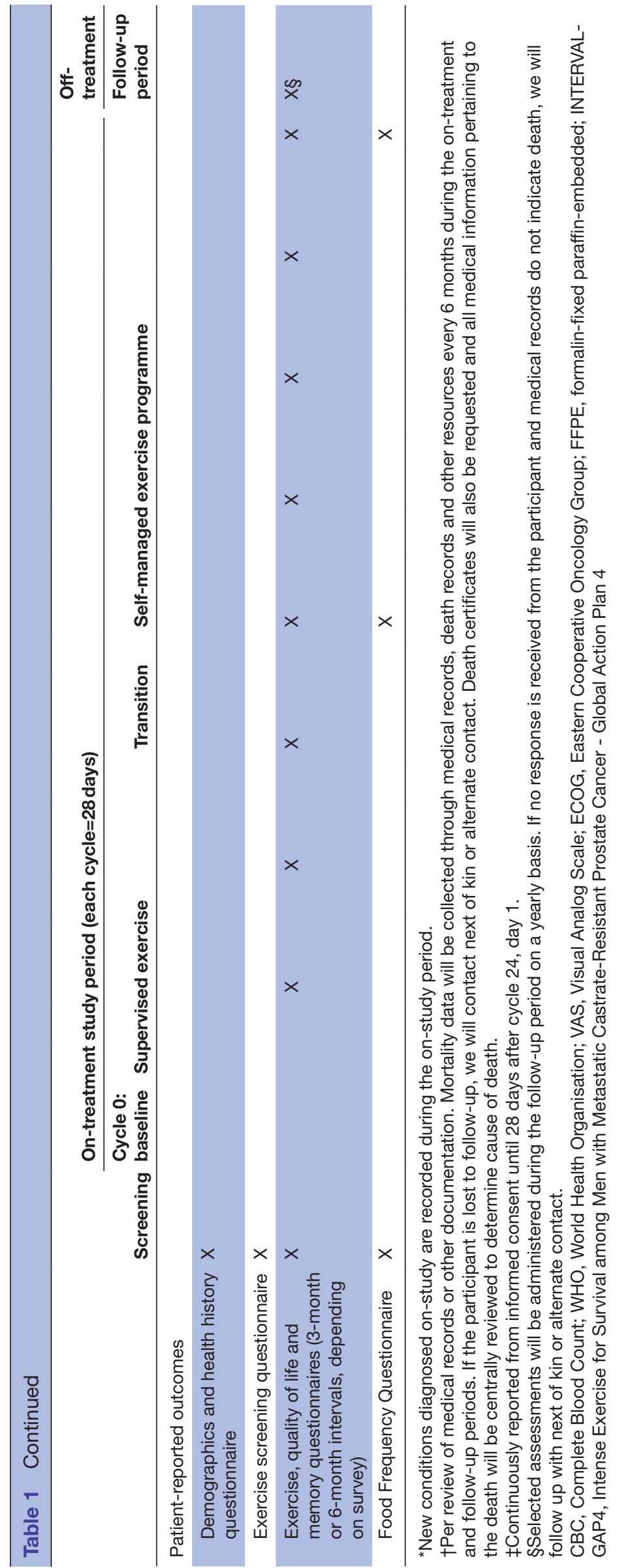

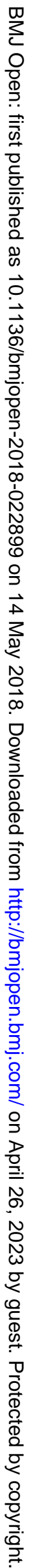


Table 2 Criteria for the establishment of disease progression following randomisation

\begin{tabular}{|c|c|}
\hline Source & Criterion \\
\hline Bone scan & $\begin{array}{l}\text { Appearance of } \geq 2 \text { new lesions on } \\
\text { bone scan, for bone scans that are } \\
\text { completed }>12 \text { weeks following } \\
\text { randomisation. }\end{array}$ \\
\hline CT/MRI scans & $\begin{array}{l}\geq 20 \% \text { increase in the sum of lesion } \\
\text { diameters, taking the reference as the } \\
\text { smallest sum on study. In addition to this } \\
\text { relative increase by } 20 \% \text {, the sum must } \\
\text { also demonstrate the following: } \\
\text { an absolute increase }>5 \mathrm{~mm} \text {, OR } \\
\text { the appearance of one or more new } \\
\text { lesions, OR } \\
\text { unequivocal progression of baseline } \\
\text { non-measurable lesions. }\end{array}$ \\
\hline $\begin{array}{l}\text { mCRPC therapy } \\
\text { initiation }\end{array}$ & $\begin{array}{l}\text { Development of an indication for } \\
\text { initiating a therapy for mCRPC after } \\
\text { randomisation, including but not } \\
\text { limited to, abiraterone, enzalutamide, } \\
\text { chemotherapy or radiation therapy. }\end{array}$ \\
\hline $\begin{array}{l}\text { Symptomatic } \\
\text { skeletal events }\end{array}$ & $\begin{array}{l}\text { Development of a symptomatic skeletal- } \\
\text { related event that must be attributable to } \\
\text { disease. }\end{array}$ \\
\hline
\end{tabular}

Progression will be defined based on Prostate Cancer Working Group 3 (PCWG-3) and Response Evaluation Criteria in Solid Tumours (RECIST) 1.1 as all other lesions, including small lesions (longest diameter $<10 \mathrm{~mm}$ or pathological lymph nodes 10 to $<15 \mathrm{~mm}$ short axis) as well as truly non-measurable lesions. All non-measurable lesions will be recorded at baseline. If patients have measurable disease, there must be overall worsening in non-measurable disease such that the overall tumour burden has increased substantially. The designation of disease progression solely on the basis of change in non-measurable disease in the face of stable disease or partial response of the measurable disease is extremely rare.

mCRPC, metastatic castrate-resistant prostate cancer; CT, computed tomography; MRI, magnetic resonance imaging.

by the treating physician based on the Prostate Cancer Trials Working Group 3 (PCWG-3) ${ }^{21}$ and Response Evaluation Criteria in Solid Tumours (RECIST) $1.1^{22}$ criteria to determine and monitor specific indications of disease progression (table 2). Time to disease progression will be measured from randomisation until the first of the following: first CT or bone scan documenting disease progression, initiation of a new therapy for MCRPC (clinical progression) or first occurrence of an SSE.

\section{Symptomatic skeletal-related events}

Time to the first occurrence of an SSE will be defined as the time from randomisation to documentation of any of the following: (1) use of external beam radiation therapy to relieve bone pain, (2) occurrence of new symptomatic pathological bone fractures excluding asymptomatic compression fractures, (3) known spinal cord compression, (4) change in antineoplastic therapy to treat bone pain or (5) surgical intervention to treat bone pain. This information will be determined through adverse event (AE) recordings, concomitant medication and treatment reviews, and patient medical record reviews.

Progression of pain, degree of pain and opiate use

Analgesic or opiate use will be assessed using the Brief Pain Inventory-Short Form (BPI-SF), the WHO Analgesic Scale and medical record review at entry with a lead-in period of $<28$ days. The WHO Analgesic Scale will be completed every three cycles (and confirmed by medical review), with the BPI-SF administered every three cycles until cycle 24 and annually thereafter.

\section{Immune status, inflammation, energy metabolism and androgen} metabolism

Fasted serum, plasma and buffy coat samples $(26 \mathrm{~mL}$ per visit) and first-void urine will be collected (with $4 \mathrm{~mL}$ of urine aliquots stored) at cycles 0, 6, 12 and 24. Serum and plasma aliquots will be used to interrogate a panel of markers associated with immune function and inflammation, such as interleukin (IL1 $\beta$, IL-2, IL-6), tumour necrosis factor- $\alpha$, adiponectin and $\mathrm{C}$ reactive protein. Energy metabolism will be investigated through markers including serum insulin, plasma glucose, C-peptide and IGF-1. Androgen metabolism will be explored through biomarkers including testosterone, dihydrotestosterone, androstenedione, dehydroepiandrosterone, 17-hydroxyprogesterone, 17-hydroxypregnenolone, sex hormone binding globulin and progesterone using mass spectrometry. The study is funded to analyse immune, inflammation, energy metabolism markers at the four collection time points, and androgen metabolism markers at cycles 0 and 6 . Samples will be stored in regional biorepositories across the globe throughout the trial in $-80^{\circ} \mathrm{C}$ biomedical freezers prior to batch analyses at the completion of the trial, for all patients who provided consent for this to occur at randomisation.

\section{Physical function}

Muscle strength will be assessed using a one-repetition maximum (1RM) test for chest press, leg press, seated row and/or leg extension, depending on the location and severity of any bone metastases (table 3), ${ }^{9} 1123$ recorded in kilograms. Functional performance will be assessed through the $400 \mathrm{~m}$ walk test, recording time to completion (in seconds) with heart rate maximum (HRmax), heart rate average (HRavg) and heart rate recovery (HRR) quantified. Aerobic fitness will be assessed through a medically supervised CPET to determine patient $\mathrm{VO}_{2}$ peak $\left(\mathrm{LO}_{2} / \mathrm{min}\right.$ and $\left.\mathrm{mlO}_{2} \mathrm{~kg} / \mathrm{min}\right)$ and maximum workload (Watts) during a successful CPET (RPE $\geq 9$, using the 10-point Borg Scale).${ }^{24}$ Physical function assessments are performed every three to six cycles across the 2-year on-trial period as previously described (table 1). ${ }^{210}$

\section{Quality of life}

Quality of life is measured through questionnaires every three cycles, including the Functional Assessment of 
Table 3 Exercise prescription for cycle 0 (weeks 1-4), a fully supervised introduction to exercise while incrementally building exercise capacity

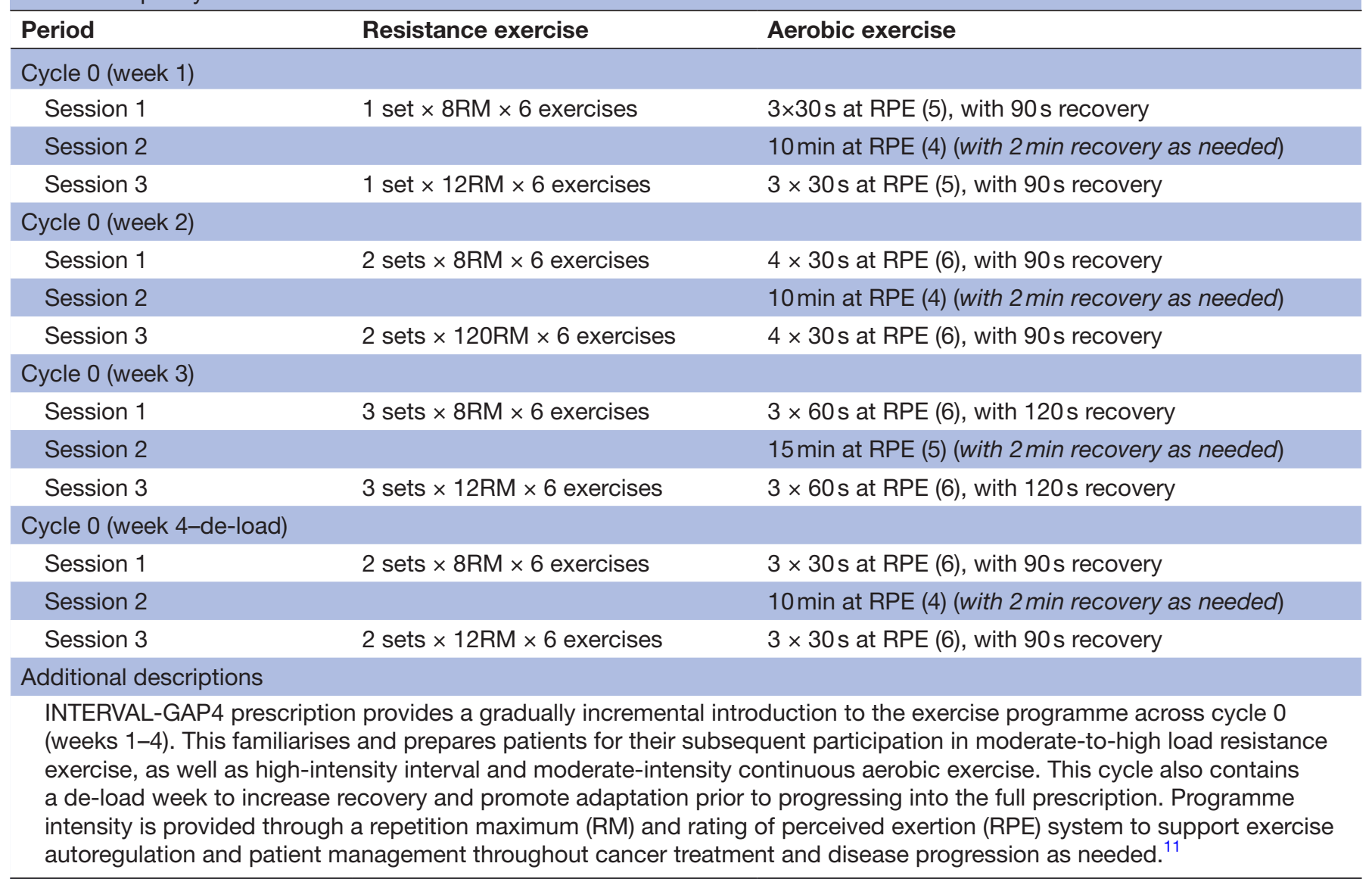

Cancer Therapy-G; Functional Assessment of Chronic Illness Fatigue subscale; European Organisation of Research and Treatment of Cancer Quality of Life Questionnaire 30; Expanded Prostate Cancer Index Composition 26; EuroQOL 5 Dimensions Questionnaire (EQ5D); State-Trait Anxiety Inventory, Center for Epidemiologic Studies Depression; and Pittsburgh Sleep Quality Index questionnaire.

\section{Programme safety}

All AEs will be graded according to the National Cancer Institute's Common Terminology Criteria for Adverse Events (V.4.0), and will be assessed at every exercise testing and training visit. AEs will also be collected in both groups once per month by telephone. AE type, severity, attribution (disease-related or exercise-related), expectedness and timing will be recorded on case report forms. Serious adverse events (SAEs) include events that may be life-threatening, require and/or prolong inpatient hospitalisation, result in persistent or significant disability or incapacity, or result in death. AEs expected on-trial include bone pain, pathological skeletal fracture, musculoskeletal injury, joint pain, falls and/or muscle soreness. All patients regardless of group will require medical clearance following AEs prior to recommencing their exercise programme.

\section{Health economics}

An economic evaluation will be performed in parallel to the trial to assess the health benefits, additional costs and potential savings of including exercise therapy as standard of care for men with mCRPC. This health economics protocol will inform the relative value for money of exercise medicine compared with other healthcare interventions in this patient population and stage of disease. Hospital resource consumption and associated costs will also be obtained to assess costs for secondary healthcare utilisation between the intervention and control groups (supervised exercise and self-directed exercise, respectively). All hospital events, including emergency department attendances and admissions, outpatient visits and procedures, and inpatient admissions for all causes will be explored to quantify and identify potential disease-related (prostate cancer) events, as well as total healthcare resource use for all other purposes inclusive of comorbidities and other chronic diseases. The cost of providing the supervised exercise and self-managed intervention will also be quantified. Due to the international distribution of investigator sites involved in this study, a regional (country by country) and global (pooled) analysis will be conducted to account for regional differences in healthcare systems, coverage and costs. 
Data on health benefits and costs will be appropriately adjusted for covariates, such as age, common comorbidities (eg, diabetes, cardiovascular disease) and body mass index. Health benefits will be measured using quality of life derived from the EQ5D and converted to a health utility scale using regional norms (where possible) to derive quality-adjusted life years for cost utility analysis. Given the duration of this multinational RCT, costs associated with health resource use and delivery of the supervised exercise intervention will be standardised to a common year. Incremental costs and benefits will be subsequently estimated and reported as an incremental cost effectiveness ratio, which will be bootstrapped ${ }^{25}$ to identify 95\% CIs. This will be subsequently used to quantify the probability of whether the intervention is good value for money, and the level of risk for patients with mCRPC not being 'better-off' by receiving supervised exercise (ie, the intervention). Deterministic sensitivity analysis will be undertaken to identify the main drivers of the costs, outcomes and value for money.

\section{Patient programmes}

\section{Exercise prescription}

Patients assigned to the intervention arm will receive a 96-week, individualised (ie, based on a needs analysis and physical assessment of patient condition and capacity), periodised (ie, the systemic organisation of exercise variation into microcycles (weekly), mesocycles (monthly) and macrocycles (annually)), progressive and autoregulated (ie, patients progress at their own pace based on variations in health, performance capability, fatigue, recovery capacity or scheduling commitments, with adjustments made each session according to patients' capacity on the day of exercise training) programme, consisting of structured resistance exercise and combinations of high-intensity interval training and moderate-intensity continuous training aerobic exercise (table 3 and 4 ). The initial 48 weeks of the programme (year 1) will be supervised in an exercise clinic setting, with a gradual tapered transition to self-management, and the subsequent 48 weeks of the programme (year 2) will be self-managed with one exercise visit required at the beginning of each cycle (every 4 weeks). This exercise prescription critically uses periodisation to maximise training stimulus and physiological adaptation while also reducing the risk of injury, overtraining or staleness, ${ }^{1126}$ autoregulation to allow patients with advanced mCRPC to self-determine their capabilities at each session collaboratively with the supervising clinical exercise physiologist, thereby lowering intensity or volume if the patient is fatigued or unwell, or raising intensity or volume if the patient is energetic and motivated. ${ }^{11}{ }^{26}$ Furthermore, the exercise programme will be modified for any patients with mCRPC with bone

Table 4 Exercise prescription for cycles 1-11 (weeks 5-48): a progressive, periodised and autoregulated programme with deload weeks, tapering supervision to self-management

\begin{tabular}{|c|c|c|}
\hline Period & Resistance exercise & Aerobic exercise \\
\hline \multicolumn{3}{|c|}{ Cycles 1-11 (week 1) } \\
\hline Session 1 & 4 sets $\times 8 \mathrm{RM} \times 6$ exercises & $6 \times 60$ s at RPE (8), with 120 s recovery \\
\hline Session 3 & 4 sets $\times 12 \mathrm{RM} \times 6$ exercises & $6 \times 60$ s at RPE (8), with 120 s recovery \\
\hline \multicolumn{3}{|c|}{ Cycles 1-11 (week 2) } \\
\hline Session 2 & & 30-40 min at RPE (6) (with 2 min recovery as needed) \\
\hline Session 3 & 4 sets $\times 10 \mathrm{RM} \times 6$ exercises & $6 \times 30$ s at RPE (9), with 90 s recovery \\
\hline \multicolumn{3}{|c|}{ Cycles 1-11 (week 3) } \\
\hline Session 1 & 3 sets $\times 8 \mathrm{RM} \times 6$ exercises & $6 \times 60$ s at RPE (8), with 120 s recovery \\
\hline Session 2 & & 30-40 min at RPE (5) (with 2 min recovery as needed) \\
\hline Session 1 & 2 sets $\times 6 \mathrm{RM} \times 6$ exercises & $4 \times 30$ s at RPE (6), with 90 s recovery \\
\hline Session 2 & & 30-40 min at RPE (4) (with 2 min recovery as needed) \\
\hline Session 3 & 2 sets $\times 10 \mathrm{RM} \times 6$ exercises & $4 \times 30$ s at RPE (6), with 90 s recovery \\
\hline \multicolumn{3}{|c|}{ Additional descriptions } \\
\hline \multicolumn{3}{|c|}{$\begin{array}{l}\text { INTERVAL-MCRPC prescription provides a periodised, progressive and individually tailored programme consisting of } \\
\text { moderate-to-high load resistance exercise, combined with high-intensity interval and moderate-intensity continuous aerobic } \\
\text { exercise. Each cycle contains a de-load week to increase recovery and promote adaptation. Programme intensity is providec } \\
\text { through a repetition maximum (RM) and rating of perceived exertion (RPE) system to support exercise autoregulation } \\
\text { throughout cancer treatment and disease progression as needed. }{ }^{11}\end{array}$} \\
\hline
\end{tabular}


Table 5 Modular, multimodal exercise programming for patients with $\mathrm{mCRPC}$ with known bone metastases across resistance, aerobic and flexibility training based on lesion sites $^{91122}$

\begin{tabular}{|c|c|c|c|c|c|c|}
\hline \multirow{2}{*}{$\begin{array}{l}\text { Metastases } \\
\text { site }\end{array}$} & \multicolumn{3}{|c|}{ Resistance } & \multicolumn{2}{|c|}{ Aerobic } & \multirow{2}{*}{$\begin{array}{l}\text { Flexibility } \\
\text { Static }\end{array}$} \\
\hline & Upper & Trunk & Lower & WB & NWB & \\
\hline Pelvis & $\sqrt{ }^{*}$ & $\sqrt{ }$ & $\sqrt{\dagger}$ & & $\sqrt{ }$ & $\sqrt{\ddagger}$ \\
\hline $\begin{array}{l}\text { Lumbar } \\
\text { spine }\end{array}$ & $\sqrt{ }^{*}$ & & $\sqrt{\dagger}$ & & $\sqrt{ }$ & $\sqrt{\ddagger}$ \\
\hline $\begin{array}{l}\text { Thoracic } \\
\text { spine/ribs }\end{array}$ & $\sqrt{ }^{*}$ & & $\sqrt{\dagger}$ & $\sqrt{ }$ & $\sqrt{ }$ & $\sqrt{\ddagger}$ \\
\hline $\begin{array}{l}\text { Proximal } \\
\text { femur }\end{array}$ & $\sqrt{ }^{*}$ & $\sqrt{ }$ & $\sqrt{\dagger}$ & & $\sqrt{ }$ & $\sqrt{\ddagger}$ \\
\hline All regions & $\sqrt{ }^{*}$ & & $\sqrt{ } \dagger$ & & $\sqrt{ }$ & $\sqrt{\ddagger}$ \\
\hline
\end{tabular}

*Exclusion of shoulder flexion/extension/abduction/adductioninclusion of elbow flexion/extension.

†Exclusion of hip extension/flexion-inclusion of knee extension/ flexion.

‡Exclusion of spine/flexion/extension/rotation.

$\sqrt{ }$, target exercise region; mCRPC, metastatic castrate-resistant prostate cancer; NWB, non-weight bearing (eg, cycling); WB, weight bearing (eg, walking).

metastases depending on the size and location of metastases (table 5), performed individually or in small groups (of up to 4-6 patients per session).

Resistance exercise intensity is prescribed using the repetition maximum (RM) method, which is monitored and adjusted throughout the programme, with weight increased or decreased as the patient becomes stronger or weaker (ie, 8RM refers to the highest amount of weight a patient can lift eight times per set). Resistance training repetitions will not be performed to the point of neuromuscular failure, but rather the set will be ceased 1-2 repetitions short of the patient being unable to complete a repetition. Performing resistance training sets to neuromuscular failure are unlikely to provide additional benefit in non-athlete populations. ${ }^{27}$

Aerobic exercise intensity is prescribed using the RPE method, where aerobic ergometer resistance or speed will be adjusted to elicit the target RPE throughout the trial. A confirmatory, supervised aerobic assessmentthe constant load test $(\mathrm{CLT})^{24}$-will be conducted at the start of each cycle to monitor aerobic fitness progression across the exercise programme including the self-management period. The CLT is a short, 3 min submaximal exercise test performed on a cycle ergometer at a preset workload (70\% of achieved CPET workload at the screening visit), with a graded 4 min warm-up and active $3 \mathrm{~min}$ unloaded cool-down (ie, $10 \mathrm{~min}$ total). This CLT workload established at baseline remains constant (unchanged) throughout the trial and is used to assess programme effectiveness with HRmax, HRavg, HRR and RPE recorded. Impact exercise is excluded from the exercise prescription for all patients as it is a contraindication for patients with bone metastases, comprising over $80 \%$ of patients with mCRPC.

\section{Behavioural support}

Patients assigned to the supervised exercise arm will also receive behavioural support to help promote programme adherence and compliance when tapering to self-management. Behavioural support is provided in text message format, the overarching focus of which will be to increase perceived control in task-specific exercises and assist patients with overcoming individual barriers to exercise, rooted in the social cognitive theory and the theory of planned behaviour constructs. ${ }^{28-30}$ The level of behavioural support provided will increase as patient self-management increases, providing one text per week (cycle 0 ), two texts per week (cycles 1-8), three texts per week (cycles 9-11) and five texts per week (cycles $\geq 12$ ). Some text messages will ask patients to provide a response in a return text message to heighten patient engagement.

\section{Psychosocial support}

Psychosocial support will be provided to all patients in the trial. Participants will be given a digital or mailed newsletter of two to three pages each month, to provide education and information on topics relevant for men with advanced prostate cancer, including, but not limited to, staying healthy, lifestyle behaviours, goal setting, managing fatigue, bone health, side effects of treatment, managing side effects, depression, social support, pain management, sexual intimacy, cognitive changes and gaining control.

\section{Self-directed exercise group (control)}

Patients randomised to the self-directed exercise group will receive the psychosocial support described above. In addition, these patients will also be provided with the current American College of Sports Medicine guidelines for physical activity for cancer survivors ${ }^{31} 32$ and print information on how to pursue a self-directed exercise programme.

This self-directed exercise strategy is being employed as it would be unethical to ask men with advanced prostate cancer to abstain from exercise for a 2-year period, owing to the documented health benefits of exercise in patients with prostate cancer with early-stage disease. Similarly, this trial uses a single-blinded study design where it is not possible to blind patients to the intervention, and given recruited patients are at the end stage of life the ability to provide supervised exercise to control patients after trial completion to optimise patient retention is not an option. Thus, it is felt the provision of printed material for self-directed exercise will assist with patient retention for men randomised to the control arm, who are free to exercise as much or as little as they like.

There is considerable contrast in the effectiveness of a supervised programme pursued in an exercise clinic versus home-based and self-directed formats; thus, we believe this design will differentiate the benefits of the exercise intervention for the primary and secondary outcomes and maintain interventional fidelity in the trial. 
To date, research into unsupervised exercise programmes in cancer populations has shown limited effectiveness. ${ }^{33-35}$

\section{Statistical considerations}

Analysis will be performed using an intention-to-treat approach ${ }^{36}$ powered to detect an HR of 0.78 for OS between patients randomised to the two treatment arms. Given a total enrolment period of 36 months, an on-trial period of $24 \times 4$ week cycles, a minimum of 36 months of follow-up after the on-treatment period; survival time following an exponential distribution; and a median OS of 33.5 months in the self-directed exercise arm, the estimated sample size required to detect an HR of 0.78 with $80 \%$ power at significance level of 0.05 is 824 (ie, 412 men in each arm). Accounting for up to $5 \%$ of patients with missing data on OS, we aim to enrol 866 men (ie, 433 men in each arm). Four interim analyses will be performed: feasibility (completed in 2016), intervention effectiveness (first $15 \%$ of patients following the six cycles using leg extension strength and $400 \mathrm{~m}$ walk test data), efficacy (first $50 \%$ of patients following death to investigate OS) and accrual assessment (quarterly analysis).

\section{Trial management}

INTERVAL-GAP4 has several levels of management to ensure the trial is appropriately governed and operational. The study protocol was established through a Protocol Development Working Group with guidance from a Steering Committee and Research Advisory Committee, each independent from each other, with globally recognised leaders in clinical and academic contexts pertaining to prostate cancer. Operationally, the INTERVAL-GAP4 trial is overseen by the Steering Committee and comanaged by the ECC (Exercise Medicine Research Institute, Edith Cowan University, Australia), the SCC (Department of Urology, UCSF, USA) and the Global Project Manager (Global Action Plan, Movember Foundation, Australia), with guidance from the Research Advisory Committee. The trial also has a Protocol Amendment Review Committee to continually evaluate protocol performance and review or approve proposed site-specific, investigator-led substudies.

INTERVAL-GAP4 has a Data Safety and Monitoring Board (DSMB) to oversee the data monitoring of the trial, and to monitor the safety of patients enrolled in the trial, which is completely independent of all other governing committees and site investigators. The trial also has a Medical Monitor Team consisting of several urologists and medical oncologists in Canada, UK and USA. The SCC oversees site training, enrolment of patients (including randomisation), study databases, clinical data collection and data auditing, behavioural and psychosocial support programmes, country-specific translations, $\mathrm{AE}$ and $\mathrm{SAE}$ reporting, liaison with the Medical Monitors and DSMB, and implementation of central data collection software (REDCap, Vanderbilt University, Nashville, USA). The ECC oversees site training of exercise physiologists and professionals pertaining to exercise programming and supervision of patients with advanced prostate cancer; oversees exercise testing and training, and delivery of the INTERVAL-GAP4 prescription; and manages exercise data collection, auditing and implementation of the exercise management software (Physitrack, Brighton, UK). All data entered into REDCap and Physitrack are de-identified (ie, using patients' study identifier), with all forms uploaded to REDCap de-identified (ie, all identifiable information is redacted).

\section{Patient and public involvement}

Movember Foundation is a charitable organisation advocating for improved outcomes for men with prostate cancer. As the funder of this global trial, with extensive consumer representative networks (ie, patients, their families and their carers) across the globe, the organisation is uniquely positioned to represent the views and experiences of consumers in Australia, Canada, UK and the USA (among others) who have engaged with Movember's activities over the past 15 years. This unique viewpoint and experience have been used to ensure that the study protocol engages participants in a respectful, ethical and impactful way, while addressing the needs of patients with mCRPC. Movember Foundation, with their consumer representative networks, will also facilitate the delivery of this study, by providing assistance with patient recruitment and support, as well as the translation and dissemination of the research findings to community members, patients and cancer support groups. In addition, the research team of this study protocol includes urologists and medical oncologists who work with the target population on a daily basis, from which these clinicians have used patient priorities, patient experience and patient preferences to help inform the development of the research questions and outcome measures. Lastly, the broader research team has conducted research studies in exercise and prostate cancer involving large quantities of participants over the course of the past 20 years, providing feedback to investigators to help design better exercise oncology clinical trials. This sizeable patient interaction across the clinical and community landscape has contributed substantially to the design of this project.

\section{ETHICS AND DISSEMINATION}

Ethics approval was first obtained at Edith Cowan University (ID: 13236 NEWTON), with a further 10 investigator sites in Australia, Canada, Europe, UK and USA since receiving site-specific human research ethics approval, prior to site activation and recruitment commencement. All future investigator sites joining the INTERVAL-GAP4 study are required to obtain site-specific human research ethics committee approvals, undergo site-based education and training, and receive a site initiation visit prior to opening for recruitment. Future amendments to the protocol and associated documentation, if any, will be deliberated and approved by the Protocol Amendment Review Committee, followed by submission to and 
approval of the Steering Committee. Approved amendments will be subsequently distributed to individual site investigators for submission to their human research ethics committees by the SCC.

Validation of exercise as medicine and its mechanisms of action will create evidence to change clinical practice. Accordingly, outcomes of this RCT will be published in international, high-quality, peer-reviewed journals, and presented at national and international conferences or research meetings. Outcomes of this study will also be delivered to community and consumer-led forums, and will be presented at local hospital departments and university seminars. Lastly, evidence derived from this RCT will be inserted into updated clinical exercise and/ or medical guidelines and position statements within national and international governing bodies.

\section{DISCUSSION}

Preliminary evidence supports the potential beneficial role of exercise for prostate cancer survival. Exercise has potential as a low-toxicity adjuvant medicine that can be combined with standard cancer therapies to improve patient outcomes,${ }^{11}$ with the exciting possibility of improving OS. ${ }^{1}$ Data from observational epidemiological studies provide a convincing body of evidence to suggest a considerable survival benefit from habitual physical activity prediagnosis and postdiagnosis in men with prostate cancer. ${ }^{437-40}$ While these studies provide useful associations, they cannot infer or establish a causal relationship, and are subject to bias due to measurement error, unmeasured and residual confounding, reverse causation, and self-selection. Consequently, there is a need for RCTs to directly evaluate the relationship between exercise (herein delivered as a tailored 2-year exercise prescription) and OS in men with prostate cancer. ${ }^{11}$ The INTERVAL-GAP4 protocol outlined in this paper aims to achieve this ambitious undertaking, and we hypothesise that a tailored, partially supervised, structured exercise intervention will deliver greater benefits than incidental or self-managed physical activity. This study is the first worldwide to explore the impact of exercise on OS in prostate cancer.

If it is demonstrated that this exercise intervention results in a clinically meaningful improvement in patient survival, then such exercise prescriptions can be immediately implemented worldwide, providing benefits to men with advanced prostate cancer. Further elucidation of the mechanisms by which exercise provides this survival benefit may inform the development of future therapeutic agents as well as improve the synergistic provision of exercise prescriptions in combination with existing therapies including chemotherapy, radiation therapy, enzalutamide and abiraterone.

Following protocol development and endorsement by the Steering Committee and Research Advisory Committee, the INTERVAL-GAP4 trial was launched in December 2015. Following trial launch, a pilot site in
Perth, Western Australia (Edith Cowan University) was chosen to demonstrate protocol feasibility across the inaugural year (recruiting from March 2016 to November 2016), whereby the study protocol went through several iterations to enable minor amendments (from version 1 to version 4) under the guidance of a Protocol Amendment Review Committee, with Steering Committee oversight. Following the established feasibility and demonstration of the study protocol (with 10 patients randomised at the pilot site in year 1), along with the concurrent establishment of each trial coordination centre (SCC, ECC), investigator sites worldwide began to open for recruitment in January 2017 in a staggered process, with six sites open midyear (July 2017) and a further five sites open by the end of the year (December 2017). The remaining investigator sites are due to open within the subsequent 12 months (December 2018) and additional sites are being considered. This study protocol conforms to the Standard Protocol Items: Recommendations for Interventional Trials and the Consensus on Exercise Reporting Template statements for RCTs and exercise reporting requirements. ${ }^{41} 42$

\section{CONCLUSIONS}

Exercise is rapidly evolving as an emerging and provocative therapy in oncology, with excellent promise to meet the broad and magnified needs of patients with advanced prostate cancer. In particular, exercise has the potential to delay disease progression and extend patient survival through numerous potential systemic and localised, mechanical and non-mechanical mechanisms. INTERVAL-GAP4 will be the first RCT to definitively examine if supervised aerobic and resistance exercise increases OS among men with mCRPC compared with self-directed physical activity.

\section{Author affiliations}

${ }^{1}$ Exercise Medicine Research Institute, Edith Cowan University, Perth, Western Australia, Australia

${ }^{2}$ School of Human Movement and Nutrition Sciences, University of Queensland, Brisbane, Queensland, Australia

${ }^{3}$ School of Medical and Health Science, Edith Cowan University, Perth, Western Australia, Australia

${ }^{4}$ Department of Urology, University of California San Francisco, San Francisco, California, USA

${ }^{5}$ Institute for Health Research, University of Notre Dame Australia, Fremantle, Western Australia, Australia

${ }^{6}$ Department of Epidemiology and Biostatistics, University of California San Francisco, San Francisco, California, USA

${ }^{7}$ Faculty of Kinesiology, Sport, and Recreation, University of Alberta, Edmonton, Alberta, Canada

${ }^{8}$ Department of Oncology and Metabolism, University of Sheffield, Sheffield, UK ${ }^{9}$ School of Medicine, Trinity College Dublin, Dublin, Ireland

${ }^{10}$ University Hospitals Bristol, NHS Foundation Trust, Bristol, UK

${ }^{11}$ Institute for Health Promotion Research, University of Texas Health, San Antonio, Texas, UK

${ }^{12}$ Department of Epidemiology, Harvard TH Chan School of Public Health, Boston, Massachusetts, USA

${ }^{13}$ Division of Gerontology and Geriatric Medicine, University of Washington, Seattle, Washington, USA 
${ }^{14}$ University of Canberra Research Institute for Sport and Exercise, University of Canberra, Canberra, Australia

${ }^{15}$ Cancer Trials Ireland, Dublin, Ireland

${ }^{16}$ Movember Foundation, Melbourne, Victoria, Australia

${ }^{17}$ Department of Medicine, University of California San Francisco, San Francisco, California, USA

${ }^{18}$ Department of Medicine, University of Minnesota, Minneapolis, Minnesota, USA

${ }^{19}$ Department of Urology, Centre Hospitalier de L'Universite de Montreal, Montreal, Quebec, Canada

Acknowledgements The authors wish to thank the many individuals worldwide who generously donate to the Movember Foundation, as it is through these philanthropic donations that our research trial has been launched. We are also indebted to all the participants in this trial, without whom none of this would be possible. The authors would also like to thank Jennette Sison, Kyle Smith, Rachelle Kirk-Burnnand, Dr Patricio Sepulveda and Paul Villanti for their time, energy and commitment to the successful operation of this study to date. We wish to also acknowledge Marijn Kortekaas, Nathan Skwortsow and Breht McConville of Physitrack Limited for their willingness to customise their exercise prescription and management software for the purposes of the INTERVAL-GAP4 trial.

Contributors All authors contributed to the design and development of the INTERVAL-GAP4 protocol. Specifically, RUN, JMC, KSC, SPF, RG, DCH, LM, SRP, SFEP, CJR and FS are members of the Steering Committee, with SAK, NHH, EMG, ELVB, OC, RUN and FS members of the Protocol Development and Protocol Amendment Review Committees. CJR, JC and FS are the study clinicians and acting medical monitors of the trial. RUN, SAK, NHH, JMC, KSC, JC, SPF, RG, DCH, LM, SRP, SFEP, EMG, ELVB, OC, MB, SG, LZ, DAG, CJR and FS collaboratively developed the study concept. RUN, NHH, SFEP, EMG, KSC and DAG designed the individualised, periodised and autoregulated exercise prescription. KSC and DCH designed and developed the behavioural support elements of the study protocol. RG and LZ produced statistical input, guidance and all calculations used in the study design. SAK, JMC and ELVB designed the data collection and management aspects of the protocol, and the psychosocial support materials for use. JC, SRP, CJR and FS established all clinical criteria, descriptions and design elements of the study. SPF, LM and OC developed the translational and biomedical components of the protocol. SAK chaired the Protocol Development Working Group (PDWG), and along with NHH cochaired the Protocol Amendment Review Committee (PARC) during the creation, optimisation and implementation of the study protocol. RUN and FS cochaired the Steering Committee, overseeing the PDWG and PARC during this process. RUN, SAK, NHH, JMC, KSC, JC, SPF, RG, DCH, LM, SRP, SFEP, EMG, ELVB, OC, MB, SG, LZ, DAG, CJR and FS contributed to writing, editing, review and approval of the study protocol, meeting the International Committee of Medical Journal Editors (ICMJE) recommendations.

Funding This trial has been internationally funded by the Movember Foundation, a global charity organisation committed to the improvement of health outcomes for men living with prostate cancer, as well as supporting men with testicular cancer and programmes focused on suicide prevention and mental health. NHH is supported by a Cancer Council of Western Australia Research Fellowship.

Competing interests MB and SG are employees of Movember Foundation, which is the funder of this research.

\section{Patient consent Not required.}

Ethics approval This study is compliant with the Declaration of Helsinki (World Medical Association) and requires human research ethics approval by the Institutional Review Board of each participating site prior to site activation. The INTERVAL-GAP4 protocol has been approved by the Movember Research Advisory Committee, the Movember Board, and has been approved by the Human Research Ethics Committees of all sites currently open for recruitment (Edith Cowan University's Human Research Ethics Committee (first), and a further 10 institutional HRECs at participating sites globally to date).

Provenance and peer review Not commissioned; peer reviewed for ethical and funding approval prior to submission.

Open Access This is an Open Access article distributed in accordance with the Creative Commons Attribution Non Commercial (CC BY-NC 4.0) license, which permits others to distribute, remix, adapt, build upon this work non-commercially, and license their derivative works on different terms, provided the original work is properly cited and the use is non-commercial. See: http://creativecommons.org/ licenses/by-nc/4.0/ (c) Article author(s) (or their employer(s) unless otherwise stated in the text of the article) 2018. All rights reserved. No commercial use is permitted unless otherwise expressly granted.

\section{REFERENCES}

1. Newton RU, Galvão DA. Accumulating evidence for physical activity and prostate cancer survival: time for a definitive trial of exercise medicine? Eur Urol 2016;70:586-7.

2. Galvão DA, Nosaka K, Taaffe DR, et al. Resistance training and reduction of treatment side effects in prostate cancer patients. Med Sci Sports Exerc 2006;38:2045-52.

3. Galvão DA, Taaffe DR, Spry N, et al. Combined resistance and aerobic exercise program reverses muscle loss in men undergoing androgen suppression therapy for prostate cancer without bone metastases: a randomized controlled trial. J Clin Oncol 2010;28:340-7.

4. Kenfield SA, Stampfer MJ, Giovannucci E, et al. Physical activity and survival after prostate cancer diagnosis in the health professionals follow-up study. J Clin Oncol 2011;29:726-32.

5. Friedenreich $\mathrm{CM}$, Wang Q, Neilson HK, et al. Physical activity and survival after prostate cancer. Eur Urol 2016;70:576-85.

6. Hardee JP, Porter RR, Sui X, et al. The effect of resistance exercise on all-cause mortality in cancer survivors. Mayo Clin Proc 2014;89:1108-15.

7. Taaffe DR, Newton RU, Spry N, et al. Effects of different exercise modalities on fatigue in prostate cancer patients undergoing androgen deprivation therapy: a year-long randomised controlled trial. Eur Urol 2017;72:293-9.

8. Ryan CJ, Smith MR, Fizazi K, et al. Abiraterone acetate plus prednisone versus placebo plus prednisone in chemotherapy-naive men with metastatic castration-resistant prostate cancer (COUAA-302): final overall survival analysis of a randomised, double-blind, placebo-controlled phase 3 study. Lancet Oncol 2015;16:152-60.

9. Beer TM, Armstrong AJ, Rathkopf DE, et al. Enzalutamide in metastatic prostate cancer before chemotherapy. N Engl J Med 2014;371:424-33.

10. Galvão DA, Taaffe DR, Spry N, et al. Exercise preserves physical function in prostate cancer patients with bone metastases. Med Sci Sports Exerc 2018;50:393-9.

11. Hart NH, Galvão DA, Newton RU. Exercise medicine for advanced prostate cancer. Curr Opin Support Palliat Care 2017;11:247-57.

12. Hart NH, Newton RU, Spry NA, et al. Can exercise suppress tumour growth in advanced prostate cancer patients with sclerotic bone metastases? A randomised, controlled study protocol examining feasibility, safety and efficacy. BMJ Open 2017;7:e014458.

13. Hawkins VN, Foster-Schubert $\mathrm{K}$, Chubak J, et al. Effect of exercise on serum sex hormones in men: a 12-month randomized clinical trial. Med Sci Sports Exerc 2008:40:223-33.

14. Matsumoto AM, Bremner WJ. Serum testosterone assays--accuracy matters. J Clin Endocrinol Metab 2004;89:520-4.

15. Stark JR, Li H, Kraft P, et al. Circulating prediagnostic interleukin-6 and $\mathrm{C}$-reactive protein and prostate cancer incidence and mortality. Int J Cancer 2009;124:2683-9.

16. Nicklas BJ, Hsu FC, Brinkley TJ, et al. Exercise training and plasma C-reactive protein and interleukin-6 in elderly people. J Am Geriatr Soc 2008;56:2045-52.

17. Zimmer $P$, Jäger $E$, Bloch $W$, et al. Influence of a six month endurance exercise program on the immune function of prostate cancer patients undergoing Antiandrogen- or Chemotherapy: design and rationale of the Prolmmun study. BMC Cancer 2013;13:272.

18. Platz EA, Till C, Goodman PJ, et al. Men with low serum cholesterol have a lower risk of high-grade prostate cancer in the placebo arm of the prostate cancer prevention trial. Cancer Epidemiol Biomarkers Prev 2009;18:2807-13.

19. Platz EA, Clinton SK, Giovannucci E. Association between plasma cholesterol and prostate cancer in the PSA era. Int $J$ Cancer 2008;123:1693-8.

20. Halabi S, Lin CY, Kelly WK, et al. Updated prognostic model for predicting overall survival in first-line chemotherapy for patients with metastatic castration-resistant prostate cancer. J Clin Oncol 2014;32:671-7.

21. Scher HI, Morris MJ, Stadler WM, et al. Trial design and objectives for castration-resistant prostate cancer: updated recommendations from the prostate cancer clinical trials working group 3. J Clin Oncol 2016;34:1402-18.

22. Eisenhauer EA, Therasse P, Bogaerts J, et al. New response evaluation criteria in solid tumours: revised RECIST guideline (version 1.1). Eur J Cancer 2009;45:228-47. 
23. Galvão DA, Taaffe DR, Cormie P, et al. Efficacy and safety of a modular multi-modal exercise program in prostate cancer patients with bone metastases: a randomized controlled trial. BMC Cancer 2011:11:517.

24. Jones LW, Eves ND, Haykowsky M, et al. Cardiorespiratory exercise testing in clinical oncology research: systematic review and practice recommendations. Lancet Oncol 2008;9:757-65.

25. Efron B. Better bootstrap confidence intervals. J Am Stat Assoc 1987;82:171-85.

26. Kraemer WJ, Ratamess N, Fry AC, et al. Influence of resistance training volume and periodization on physiological and performance adaptations in collegiate women tennis players. Am J Sports Med 2000;28:626-33.

27. Martorelli S, Cadore EL, Izquierdo M, et al. Strength training with repetitions to failure does not provide additional strength and muscle hypertrophy gains in young women. Eur J Transl Myol 2017;27:6339.

28. Trinh L, Plotnikoff RC, Rhodes RE, et al. Correlates of physical activity in a population-based sample of kidney cancer survivors: an application of the theory of planned behavior. Int J Behav Nutr Phys Act 2012;9:96.

29. Karvinen KH, Courneya KS, Campbell KL, et al. Correlates of exercise motivation and behavior in a population-based sample of endometrial cancer survivors: an application of the Theory of Planned Behavior. Int J Behav Nutr Phys Act 2007;4:21.

30. Basen-Engquist K, Carmack CL, Li Y, et al. Social-cognitive theory predictors of exercise behavior in endometrial cancer survivors. Health Psychol 2013;32:1137-48.

31. Schmitz KH, Courneya KS, Matthews C, et al. American College of Sports Medicine roundtable on exercise guidelines for cancer survivors. Med Sci Sports Exerc 2010;42:1409-26.

32. Wolin KY, Schwartz AL, Matthews CE, et al. Implementing the exercise guidelines for cancer survivors. J Support Oncol 2012;10:171-7.
33. Galvão DA, Spry N, Denham J, et al. A multicentre year-long randomised controlled trial of exercise training targeting physical functioning in men with prostate cancer previously treated with androgen suppression and radiation from TROG 03.04 RADAR. Eur Urol 2014;65.

34. Morey MC, Snyder DC, Sloane R, et al. Effects of home-based diet and exercise on functional outcomes among older, overweight longterm cancer survivors: RENEW: a randomized controlled trial. JAMA 2009;301:1883-91.

35. Baumann FT, Zopf EM, Bloch W. Clinical exercise interventions in prostate cancer patients--a systematic review of randomized controlled trials. Support Care Cancer 2012;20:221-33.

36. Gupta SK. Intention-to-treat concept: a review. Perspect Clin Res 2011;2:109-12

37. Richman EL, Kenfield SA, Stampfer MJ, et al. Physical activity after diagnosis and risk of prostate cancer progression: data from the cancer of the prostate strategic urologic research endeavor. Cancer Res 2011;71:3889-95.

38. Peisch SF, Van Blarigan EL, Chan JM, et al. Prostate cancer progression and mortality: a review of diet and lifestyle factors. World J Urol 2017;35:867-74.

39. Kenfield SA, Batista JL, Jahn JL, et al. Development and application of a lifestyle score for prevention of lethal prostate cancer. $J$ Natl Cancer Inst 2016;108:108

40. Bonn SE, Sjölander A, Lagerros YT, et al. Physical activity and survival among men diagnosed with prostate cancer. Cancer Epidemiol Biomarkers Prev 2015;24:57-64.

41. Chan AW, Tetzlaff JM, Altman DG, et al. SPIRIT 2013 statement: defining standard protocol items for clinical trials. Ann Intern Med 2013;158:200-7.

42. Slade SC, Dionne CE, Underwood M, et al. Consensus on Exercise Reporting Template (CERT): explanation and elaboration statement. Br J Sports Med 2016;50:1428-37. 